\title{
DAM and the art of implementing a central brand strategy in a decentralized organization
}

\section{Joost Schriever}

is International Marketing Manager at Randstad Holding. He joined the company in 1980 and truly knows it inside and out, having been a branch manager, product manager and director of External Relations, among other roles. Together with a colleague, he is now responsible for marketing resource and brand identity management for the Randstad brand worldwide.

\section{Keywords: marketing, branding, strategy, process, communication}

Abstract More and more organizations are discovering the value DAM adds to their branding and marketing execution. DAM-based tools such as online brand and house style guides, perhaps with an image library and some downloadable materials, are becoming commonplace. Yet our experience at Randstad is that DAM can be and do so much more when you treat it not as a database or IT system but as a process tool or way of working - a kind of Office Suite for marketeers. By enabling us to implement a single, global brand in a local, decentralized organization, DAM enables us to achieve a higher return on investment than we otherwise could. Journal of Digital Asset Management (2007) 3, 124-129. doi:10.1057/palgrave.dam.3650079

\section{INTRODUCTION}

Randstad introduced DAM in 2002 as part of a move to Marketing Resource Management (MRM). Starting small, we kept adding to the system with one goal in mind: to make it the "must have" tool that our marketeers need to carry out their marketing work.

This approach - treating DAM as the backbone of a new operational process means that today, we can store and retrieve digital information and can also use it to create a whole range of marketing assets.

\section{A CAN-DO MENTALITY}

Before I go into how we have implemented and use DAM at Randstad, let me give you a bit of context that will help explain the choices we made - especially regarding the implementation of DAM.

The first thing that you need to know is that, as a staffing and HR solutions company, Randstad really is a people-driven business. Our values are "to know, to serve and to trust" our clients, our candidates/flexworkers and each other with the aim of creating the best match between client and candidate/flexworker. And we are in the top three in our field with, at the time of writing, 2,600 offices in 20 countries in Europe, North America and Asia.

Culture-wise, Randstad is a service organization, which means we need staff who can think on their feet and make decisions. We have good processes, but we are not a process company. In terms of both operating structure and mentality, we are a decentralized company that believes that the people in our country organizations know what is best in their market.

\section{FROM GLOBAL TO LOCAL AND BACK AGAIN}

This context had clear implications when, in 2002, we decided that we needed to replace our "every country does its own thing" approach to communication, to a single, unified brand strategy. This would involve introducing one brand positioning and accompanying communication concept for all the countries we operate in.

This represented a major change. Although Randstad had employed a single brand positioning in the past, this had disappeared in the 1990s when the company strategy changed from one of organic growth and Greenfield start-ups, to acquisition-driven expansion. 
An unforeseen side-effect of this was that a previously cohesive organization became a collection of largely stand-alone businesses, each with its own culture, brand positioning and approach to the market. They operated under the Randstad name, but in reality the company was no longer acting as one brand. Although this had certain local advantages, these were outweighed by the expense of supporting local-for-local communications. In addition, there was the issue of international clients, who experienced a different Randstad in different countries.

\section{THE NEED TO PERSUADE}

The new brand strategy focused on building the Randstad name internationally rather than via local sub-brands and labels. This was logical given Randstad's international growth ambitions, but logic does not always win the argument. As most organizations have found, no matter how good the proposal is, it does not take long for the "yes, but" brigade to get busy.

In our case, we foresaw another reason for potential resistance: the fact that our new brand strategy involved a significant change in the role our marketeers fulfill.

Before the new brand strategy, local marketeers were responsible for their own marketing and branding (based loosely on a common house style); now they would be responsible for adapting a central, global concept for local use. You could argue that we were de-skilling their role, though I see it as taking care and optimizing the basics so they have more time and resources to concentrate on innovative marketing. Yet at the same time as standardizing around a global concept, we also wanted them to continue to exercise ownership over their local communications and to develop new ideas and share best practices. We wanted just them to do this based on the materials developed at the center. It would not be an easy sale to make.

\section{CONTROL OR SUPPORT?}

From a theoretical point of view, the simplest way to control and implement our new brand strategy would have been to say "this is it" and then run it from headquarters. The reality was that in a decentralized organization, this was never going to fly unless we spend a huge amount of time and effort on restructuring our marketing organization, which could only come at the expense of getting on with implementing the new brand strategy.

The smart option, therefore, was to find a way to capture the efficiency and control benefits of centralization within the existing, decentralized structure.

\section{DAM - IT SYSTEM OR WORK PROCESS TOOL?}

MRM looked like it could provide the answer. MRM is a framework for managing marketing resources, of which DAM is one element. Today our MRM framework consists of two international marketing managers (me and a colleague), a brand team of senior marketing executives that meet every six weeks, an international marketing meeting that we hold twice a year, a helpdesk for day-to-day questions - and a DAM system.

In DAM, we saw an opportunity to align our brand internationally while retaining our existing, decentralized structure. To do so, however, we would need to find a DAM system that was more than a database but which could be developed into a process tool, DAM should be a sort of Office Suite for marketeers. It would provide a centrally developed palette of tools and options that would enable our marketeers to create locally suitable yet globally aligned advertising and marketing communications.

\section{START SIMPLY AND THEN EXPAND}

The result is Randstad ID, a DAM system developed for us by NykampNyboer and IT specialist Capital ID. NykampNyboer specializes in visual performance management and what attracted us to them was that they also see the challenge of DAM as lying not in the IT but in integrating DAM into the overall brand strategy and processes.

One immediate result was that rather than going for a "Rolls Royce" solution that offered more functionality than we would have known what to do with, we started slowly, getting people used to the idea, learning what we needed and adding new functionalities as we 
went. ID is a modular solution; hence, if we see a new opportunity that looks interesting or the time is simply right to add something new, we can do it.

It followed from this that ID was simply an extranet for Randstad marketeers and suppliers involved in the brand project. Then, as the launch of "Good to know you" approached, we expanded its role with the addition of an online brand book. This explained the new brand strategy, the communication concept, the brand architecture and so on. This was set in the context of Randstad's history and outlined the thinking behind "Good to know you" and the look and feel of the concept.

As the communication concept has expanded, we have added a house style guide (letterheads, logos, business cards, etc) and a guide on how to apply the "Good to know you" concept in our branches. This covers the standard furniture, decorations, lighting and other elements that are available. Thanks to the switch to a single concept and standardization, we have reduced the number of suppliers, with all the economies of scale this implies. Most recently, we added a guide to visual communication in the branches - how to position posters, job cards and the like, taking account of different window sizes, building materials and so on.

\section{DIGITAL-BASED MARKETING EXECUTION}

While a huge step forward (no more printing, easy updates), placing guidelines and catalogs online does not in itself change the way marketing is done. Hence, as people became more familiar with the brand concept and started to feel a sense of involvement and responsibility for its implementation, we started to add new functions that would turn ID into a "brand toolbox" — something people would use every day to do their jobs. For us, this was the key to solving the issue on how to introduce and manage a central brand concept in an individualistic, decentralized organization.

As a result, ID today is a digital database, a knowledge-sharing tool, a productivity tool and an online store in one. For example, ID is now home to 750 multi-language files, mainly consisting of templates and wizards in each of our local languages. With these, every Randstad marketeer can create adverts, brochures, window posters, business cards, stationery and more, safe in the knowledge that everything they need is readily available and approved (and if it is not available, they can contact a special help desk).

We now also have a database containing 500 photographs, all commissioned by Randstad (so we own the copyright), all in line with the "Good to know you" concept, and all available for our marketeers to use. Another feature we have added is a best practices section so we can learn from our colleagues in other countries, adapting and re-using where it makes sense. And there is now also a web shop, where you can order marketing and promotional materials, plus items out of Randstad's branch fixtures and fittings catalog.

Through this, ID really has grown into the brand toolbox we foresaw at the beginning, taking it to the point where it becomes an integral and unavoidable part of our marketing execution.

\section{FACILITATING GROWTH}

Another by-product of ID is that it has made it easier for us to grow. In recent years, we have entered India, China, Turkey and Japan, and we continue to focus on growing our presence in less-mature flexwork markets, such as Italy and Spain. In some cases we have taken over and rebranded a local organization, in others we start from scratch, while in developing flexwork markets, we are typically engaged in both expanding our presence and winning over people to the flexwork concept.

We find that MRM is of considerable help in each of these scenarios. For instance, it takes far less time and far less money to convert an acquired company into a Randstad company when all it involves is going to ID's online shop and ordering the necessary fixtures and fittings. There is no need to find local suppliers, the costs are lower and it is far quicker. The only extra thing that you will probably need is local photography. It also helps to be able to show people how their working environment will look.

Likewise, ID makes it quicker and easier to create marketing collateral such as posters, brochures, job ad cards and so on. And while it 
is true that being web based can be a drawback in parts of Asia, because the internet is unreliable there, there are ways around this. For China, for instance, we have put a copy of ID onto a laptop that is not connected to the internet. It is not ideal, but it does work.

In countries like Spain and Italy, we have found that the introduction of an international brand concept has helped not only to build the Randstad brand and share of mind, but also to improve people's perception of the whole sector. In Spain, for example, flexwork and temporary employment agencies are seen as something for students or people looking for a simple job for a short period. Visit the Netherlands, however, and they will see that professionals also work via Randstad because they recognize that we provide more variety and more career options and opportunities than a "normal" job.

\section{PUBLISHING-ON-DEMAND}

Now that ID's role is understood and firmly established within the MRM mix, we are finding new areas into which we can extend our use of DAM.

Already, we use it for printing-on-demand within the local branches, typically for everyday marketing tasks such as making window posters, running of job-vacancy cards and the like. We have, however, seen even bigger opportunities change the way we work.

In Germany, for example, we have been running a pilot project in which our consultants use a new publish-on-demand module to create recruitment ads that will run in local newspapers, our own vacancy site and thirdparty sites like Monster.com. With this, a marketing manager can create an ad just by working through a series of wizards. These help him/her to decide the type of ad that is needed, the colors to use, the number of columns, the image that should go with it, which version of the Randstad logo to use and so on. All this is done within the ID environment.

In addition to creating the ad, however, ID also enables the manager to do everything that used to be done by outside agencies, everything from scheduling which media to target and getting a cost estimate, to generating and inserting the ad into the newspaper or magazine's publishing calendar. No more calling the ad agency to make a new ad, DTP it, proof it and so on. And no need to use a mediabuying agency or ad agency. That is bad news for the media and ad agencies, but it reduces costs for Randstad. This new function is now out of the pilot phase in Germany — we have made about 500 ads with it so far - and we plan to introduce it in Spain, Italy and Belgium shortly.

\section{THE BOTTOM LINE}

Five years since we began and it is fair to say that the impact of MRM and DAM on Randstad's branding has been bigger and more far-reaching than anyone expected it to be. Less than a year after we implemented "Good to know you," a look in our brand room or a walk down the high street confirmed that we were starting to talk with one voice and to present the brand consistently. And while a few of our marketeers decided that adapting company-wide materials rather than developing their own was a step too far, the vast majority have seen it as an opportunity to concentrate on real, flesh and blood marketing.

Today, Randstad operates as one brand. There are variations and interpretations, as there should be, but we clearly speak with one voice. Organizationally, efficiency has gone up while, process-wise, the time between decision and implementation has been cut and the impact increased, thanks to the introduction of ID.

Financially, we have reduced our creative and design costs by 30 per cent, reduced traffic and media-bureau costs by 20 per cent, reduced the cost of distributing marketing materials by 100 per cent and reduced our printing costs by 25 per cent. We estimate that moving to publishingon-demand could reduce our media-buying costs by 30 per cent.

This money has been used to fund more marketing and advertising, which has undoubtedly helped fuel the rapid growth of recent years, which has seen us increase our turnover and expand into new growth markets like India, China and Turkey.

Figures 1 and 2 show the situation before and after global branding. 


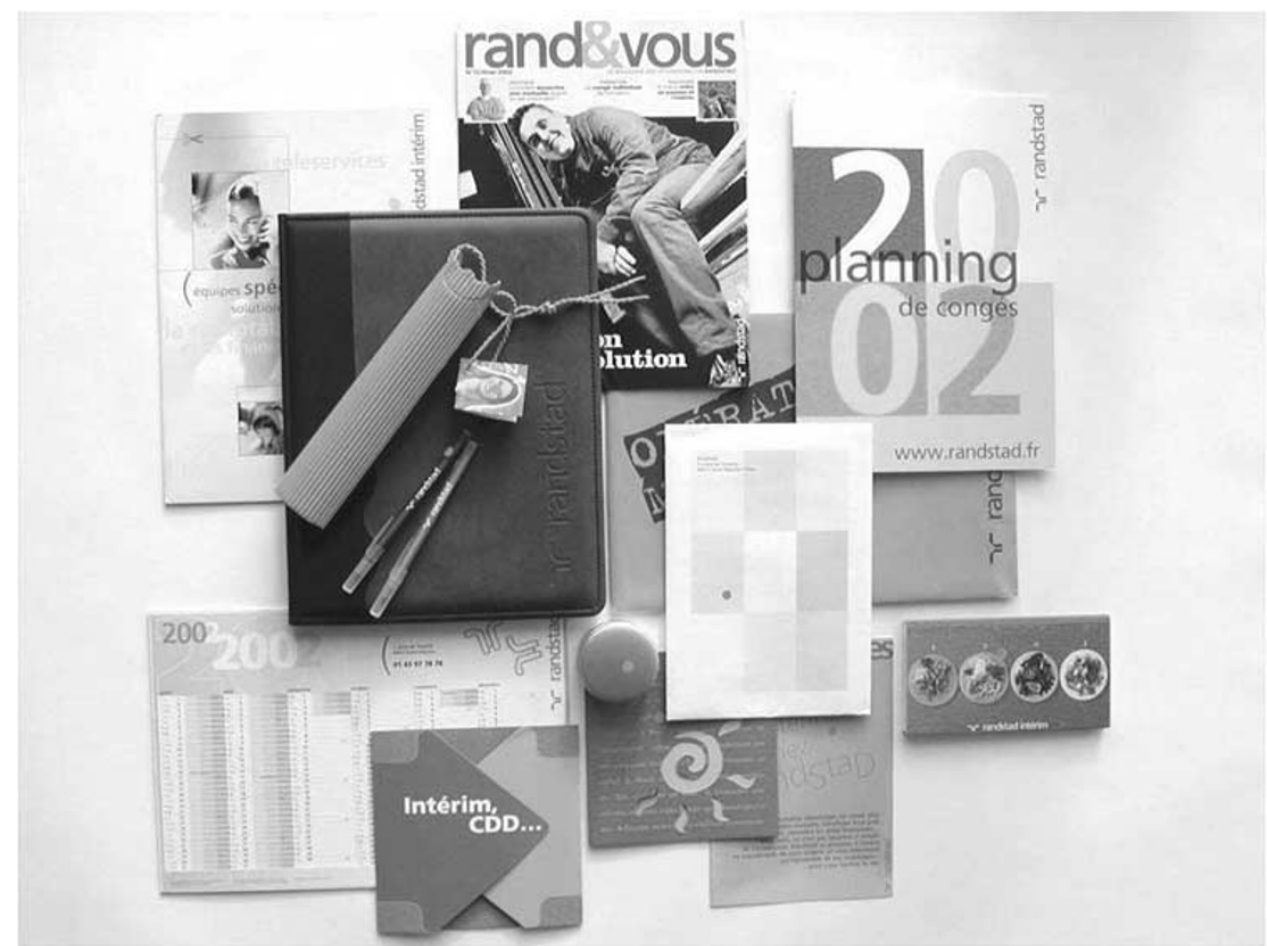

Figure 1: The situation before global branding

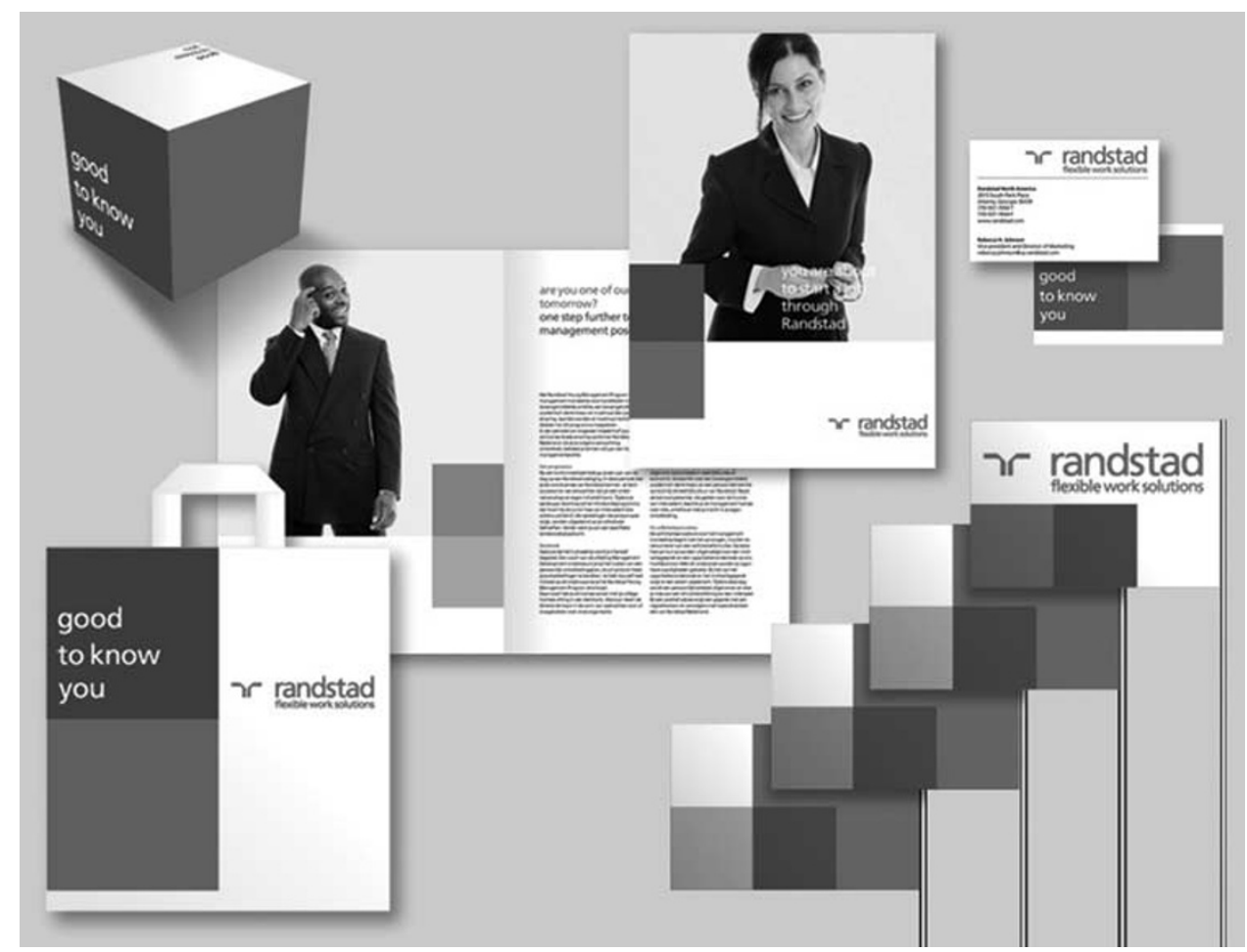

Figure 2: The situation after global branding 


\section{CONCLUSION}

Looking at where we stand now, I believe that the success of DAM in helping us build the brand and grow our businesses is because we treat it as way of working rather than as an IT solution.

While ID is based on the idea of using centrally created assets, its development is geared to the idea that we are supporting every one of our marketeers in his/her work - not constantly looking over their shoulder and telling them what to do. The wizards, templates, online store, image library and house style elements give you a degree of control and choice in how you implement the concept. In the end, each marketeer decides how a brochure, poster, vacancy ad or other marketing tool will look, within the framework of the overall concept. If you place all the materials side by side, you will see the differences - the US tends to use colors more strongly than Belgium, say - but you will also see a living, breathing brand that is vividly and recognizably the same while also being in tune with local conditions and traditions.

Because of this, we have succeeded in aligning or introducing the Randstad brand in 20 countries just by providing the assets, tools, workflows and process steps our marketeers need to get the job done. I firmly believe that in a decentralized organization like Randstad, we would never have come so far or so quickly without employing DAM as the basis for a new way of working. 